\title{
THE BIPOLAR-FLOW PHENOMENON
}

\author{
Wolfgang Kundt, \\ Institut für Astrophysik der Universität Bonn \\ Auf dem Hügel 71 \\ 53 Bonn 1 \\ Federal Republic of Germany
}

ABSTRACT: Very young stars, known as 'pre-T-Tauri stars' (PTTS) or 'young stellar objects' (YSO), tend to be surrounded by elongated outflow regions involving 'HerbigHaro objects' (HH). Such 'bipolar flows' (BF) are reminiscent of the extragalactic radio sources for which a consensus has formed in 1986 that their jets consist of extremely relativistic pair plasma, of typical Lorentz factor 1041 , generated by an 'active galactic nucleus' (AGN); [refs 6,7]. Here I collect new circumstantial evidence for the relativistic nature of $\mathrm{BFs}$.

In fact, the relativistic nature of the jet plasma of stellar BFs has been derived, in ref 2 , from both particle number and momentum conservation in the head of the flow where the jet turns subsonic across a terminal shock. A most plausible way of generating a relativistic flow is through localized magnetospheric discharges near an extremely spinning, strongly magnetized object; the pair plasma results via collisions of the charges with stellar photons once the discharge energy exceeds threshold (of order $10^{12} \mathrm{eV}$ ). This criterion applies equally to all four families of bipolar flows, viz, to YSOs, AGN, young binary neutron stars, and young binary white dwarfs (planetary nebulae) [ref 6]. The magnetospheric pair plasma is pumped centrifugally into a surrounding overpressure bubble [ref 3] which discharges in the form of two narrow, antipodal jets; fig. 1 . In this way, the central engine combines the properties of an ordinary star with those of a fast, magnetized rotator.

The predicted relativistic nature of the BFs driven by YSOs has not easily appealed to the scientific community because of the lack of evidence: One observes low-ionization emission lines from 'slowly' moving HHs $\left(v \leqslant 10^{-3} c\right)$. On the other hand, freely propagating pair-plasma jets are thought 


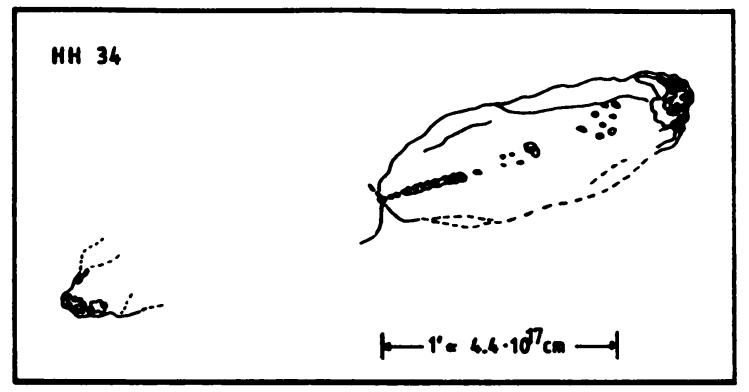

Fig.1:Optical CCD contour map (drawn) of $\mathrm{HH} 34$; from Bührke et al: A \& A $\underline{200}, 99(1988)$.

to be loss-free; most of the observed radiation comes from the impacted, heavy channel-wall and bowshock material which has absorbed part of the jet momentum. Nevertheless, the radio jet of L $1551 /$ IRS 5 shares a spectral index of $\alpha=0.3$ with the synchrotron sources in several AGN [refs $1 ; 6,7]$, and so do the (jet) sources HH 1-2, S 68 (Serpens), NGC 2071, G 192.58-0.04, and (probably) the polarized radio double $S$ 187 [refs 8,9] as well as an HH-like 'streamer' near $V 571$ Ori [ref 10]. Even more striking is the finding, in ref 5 , of strongly non-thermal radio emission from three $T$-Tauri stars whose size is a few times stellar: TTSs are understood to descend from PTTSs, hence to be spun down and less magnetic compared with the former!

Another observation which proves the occasional narrowness of the outflows from YSOs is the UV variability of $\mathrm{HH} 29$, the moving emission knot at a distance of $1017.6 \mathrm{~cm}$ from L1551/IRS 5. According to ref 4, HH 29 has varied by a factor of 4 within five months. The authors conclude at a size of the emission region of $\leqslant 10 \mathrm{AU}$, some 10-3.s times the distance from the central engine! clearly, such extreme focussing asks for an essentially weightless jet substance.

\section{References:}

1. Bieging, J.H. Cand Cohen, M.: Ap.J. 289, L5 (1985)

2. Blome, H.J. and Kundt, W.: Astroph. Sp.Sci. 148, 34 (1988)

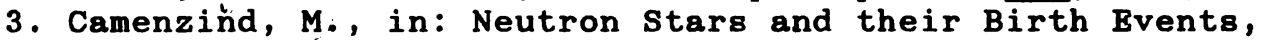
NATO ASI C 300 , ed. W. Kundt, Kluwer 1990.

4. Cameron, M. and Liseau, R.: UV-observs. of HH obj. associated with bipolar molecular outflows: A \& A, in press

5. Feigelson, E.D., Lonsdale, C.L. and Phillips, R.B.: AAS 20.07 , Jan. 1990

6. Kundt, W.: Astrophysical Jets and their Bngines, NATO ASI C 208, Reidel 1987 .

7. Kundt, W.: The Galactic Center, Astroph. Sp.Sci., in press

8. Rodriguez, L.F., et al: Ap.J. 346, L85 (1989)

9. Snell, R.N. and Bally, J.: Ap.J. $\underline{303}, 683$ (1986)

10. Yusef-Zadeh, F., et al.: Ap.J. 348, L 61 (1990). 